DOI: $10.17725 /$ rensit.2019.11.299

\title{
About AlGaAs-heterostructures CVC kinetics simulation
}

\author{
Natalia A. Vetrova, Evgeny V. Kuimov, Sergey A. Meshkov, Vasily D. Shashurin \\ Bauman Moscow State Technical University, http://bmstu.ru/ \\ Moscow 105005, Russian Federation
}

E-mail: vetrova@bmstu.ru,ekjmo@mail.ru,sb67241@mail.ru,schashurin@bmstu.ru

Received 21.08.2019, peer reviewed 20.09.2019, accepted 23.09.2019

\begin{abstract}
A model of degradation of AlGaAs-heterostructures is presented. The model of dissipative processes was extended to the case of a diffusion-blured aluminum profile. The definition of the $\Gamma$-X-mixing operator is generalized for potentials without explicit heterojunctions. The effect of degradation processes on inelastic scattering is taken into account by applying the diffusion equation to the optical potential profile. The self-consistency algorithm was optimized in order to reduce the calculation time. This paper proposes a faster method based on the reduction in the number of calculated integrals for electrons density. A number of test structures were simulated via developed algorithm. The deviation of the calculation results from the experimental data on the curvature of CVC initial section does not exceed 3\%. Thus, we can conclude that it is expedient to use the model to calculate the kinetics of heterostructures CVC initial section at elevated temperatures, including within the framework of the task of predicting the GIC and MIC UHF reliability indicators based on multilayer AlGaAs-heterostructures.
\end{abstract}

Keywords: heterostructures, nanoelectronics, mathematical modeling, structure degradation, selfconsistent potential, dissipative processes.

UDC 004.052, 538.91

Acknowledgments: This work was financially supported by the Ministry of Education and Science of the Russian Federation, project No. 16.1663.2017 / 4.6.

For citation: Natalia A. Vetrova, Evgeny V. Kuimov, Sergey A. Meshkov, Vasily D. Shashurin. About AlGaAsheterostructures CVC kinetics simulation. RENSIT, 2019, 11(3):299-306; DOI: 10.17725/rensit.2019.11.299.

\section{Contents}

1. INTRODUCTION (299)

2. StruCture DEgRagation (300)

3. Dissipative processes (301)

4. Self-consistent potential (302)

5. Results (304)

6. Conclusion (304)

REFERENCES (305)

\section{INTRODUCTION}

Today, one of the most important design issues for nanoelectronic devices based on $\mathrm{AlGaAs}$-heterostructures is the prediction of their reliability indicators. Firstly, such devices are promising for use as the basis for ultrafast devices of a new generation [1,2] in industries related to high requirements for reliability $[3,4]$. Secondly, a high cost of testing and the time constraints on their implementation makes physical and mathematical simulation of aging devices [5] more attractive compared with a classical statistical method of the theory of reliability tests.

Degradation of AlGaAs-heterostructures is attributed [6] to diffusion processes in semiconductor layers. A profile of molar fraction of aluminum is blurred under effect of high temperatures, which affects the potential energy of electrons and a position of metastable levels. The concentration profile of silicon (a donor impurity in the contact layers) is blurred too [7]. Also, metals of the contacts and contact areas penetrate into the layers of the heterostructure over time, 
which leads to an increasing of resistance of contact areas [8].

Existing mathematical models of current transfer in AlGaAs-heterostructures are based on the assumption that the transitions between the layers are localized. A calculation of the tunnel transparency coefficient within framework of the formalism of wave functions is easily generalized to the case of a continuous profile by replacing a stepped profile of potential energy with a continuous one. However, models of dissipative processes $[9,10]$ require coordinates of the heterointerfaces be explicitly specified, which is impossible to do in the case of the diffusion-blurred profile of the molar fraction of aluminum. Consequently, such models are not suitable for current transfer simulations with taking into account the diffusion blurring of a profile of aluminum.

Thus, the task of predicting the reliability of devices based on $\mathrm{AlGaAs-heterostructures}$ includes the following subtasks: structure degradation simulation (i.e., diffusion blurring of impurity profiles and resistance growth of contact areas calculation) and generalizing current transfer models to the case of structures with a continuous impurity profile along with optimization self-consistenting procedures.

A numerical calculation of the current-voltage characteristics (CVC) of heterostructures is characterized by a high time complexity, which causes a problem with kinetics of CVC simulation because of repeated self-consistent calculation. The most resource-intensive part of the calculation algorithm is the step of calculating the electron concentration within the calculation of the self-consistent potential [11,12]. Optimization of this procedure allows to reduce the calculation time for both a single CVC and their kinetics.

\section{STRUCTURE DEGRADATION}

In this paper, the model of structure degradation is based on the position of the dominant role of diffusion in degradation processes. Thus, the degradation of AlGaAsheterostructures in the framework of the models presented is due to the diffusion of aluminum and silicon, as well as the penetration of metal from ohmic contacts into semiconductor contact layers, which causes their resistance increases.

The diffusion of aluminum and silicon can be described using the Fick equation [13]. As the boundary conditions, the condition of equality to zero of the substance flow at the boundaries of the simulated region was chosen, and the step profile approximation was taken as the initial condition. The diffusion coefficient was estimated using the Arrhenius equation with activation energy of $3.5 \mathrm{eV}$ and a pre-exponential factor of $0.17 \mathrm{~cm}^{2} / \mathrm{s}$ [8].

Fick's equation was solved using the finite difference method. In this case, one grid was used to simulate diffusion and calculate the electron wave functions to avoid loss of accuracy when interpolating grid values of impurity concentrations. To solve this problem, elements of finite difference equations are obtained by averaging the terms of the Fick equation on the grid cells. The result of solving the obtained equations is the average fractions of aluminum and the average concentrations of silicon in the grid cells. These quantities were later used to construct a finite difference Hamiltonian, which allows not to construct a separate grid for solving the Schrödinger equation. 

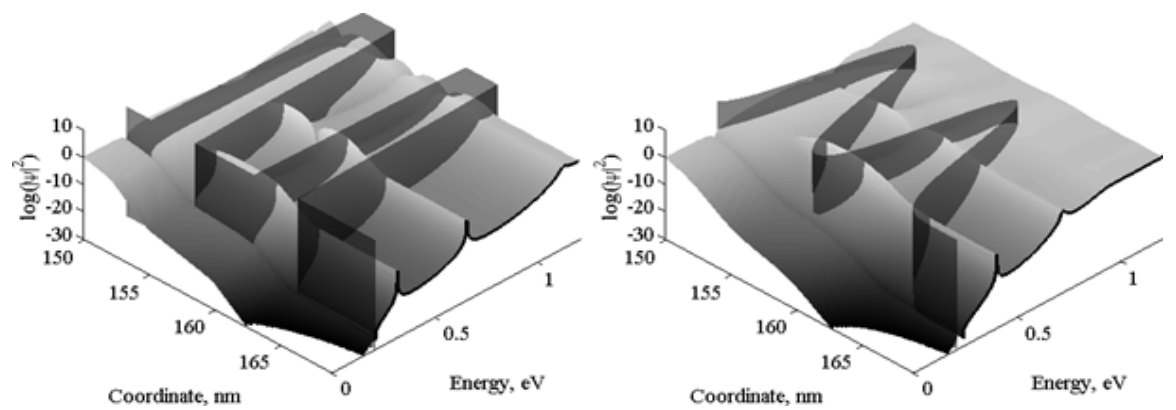

Fig. 1. The electron density logarithm before (left) and after (right) annealing. Black surfaces - the profile of the potential energy of an electron.

To estimate the effect of structure degradation, technological annealing of a resonant tunneling structure (RTS) was simulated at a temperature of $800^{\circ} \mathrm{C}[14$ 17]. The duration of annealing is $30 \mathrm{~s}$, but in order to make the calculation results more distinguishable, the simulated annealing time is increased 4 times. The diffusion processes of aluminum and silicon were simulated. The results of calculating the electron density and the tunnel transparency coefficient are shown in Fig. 1. The wave functions were calculated at zero voltage in the self-consistent field approximation [11].

As can be seen from the figures, diffusion blurring leads to a shift of the resonance levels to high energies, as well as the broadening of the resonance levels. This leads to an

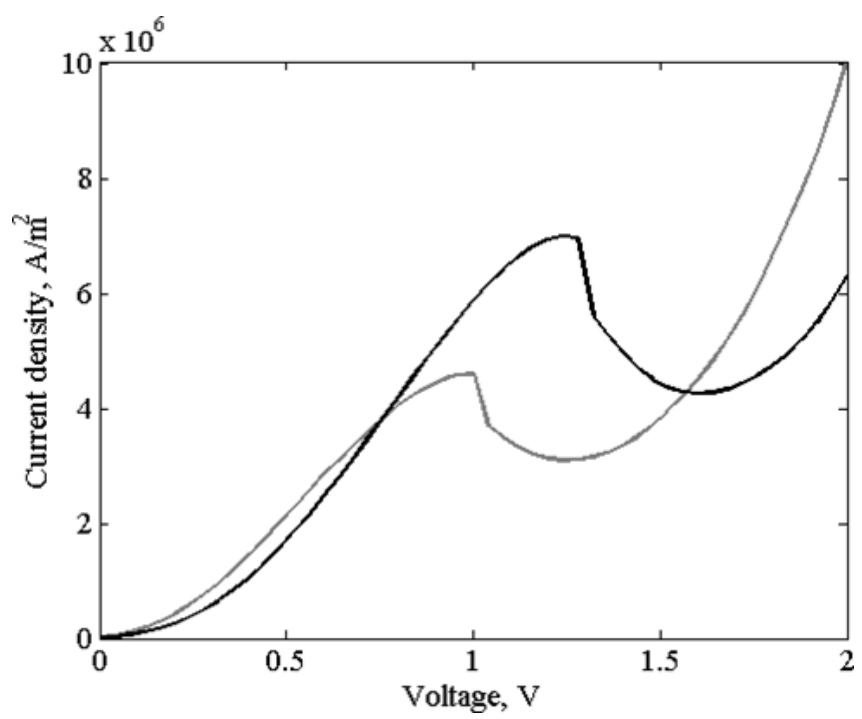

Fig. 2. CVC RTS before (grey line) and after (black line) annealing. increase in peak voltage and peak current, in addition, to an increase in the slope of CVC characteristic, as shown in Fig. 2. The duration of annealing in calculating CVC characteristic in Fig. 2 corresponds to the duration of the simulated technological process and is $30 \mathrm{~s}$.

\section{DISSIPATIVE PROCESSES}

The model of current transfer includes two types of electron scattering — intervalley scattering at heterointerfaces [9] and inelastic scattering by phonons [10]. The inter-valley scattering is taken into account by the two-channel model, and the scattering on phonons is taken into account using the optical potential method. In $[9,10]$ models of dissipative processes were developed for the case of precise heterointerfaces. However, it is difficult to determine the positions of heterointerfaces, when taking into account diffusional changes in the aluminum profile; therefore, these models require generalization. The motion of electrons in the two-channel model is described by the Schrödinger equations for an open system, in which a communication operator is included that describes the mixing of electronic states in the channels

$$
\left(\begin{array}{cc}
H^{\Gamma}-E+\Sigma^{\Gamma} & A \\
A & H^{X}-E+\Sigma^{X}
\end{array}\right)\left(\begin{array}{l}
\psi^{\Gamma} \\
\psi^{X}
\end{array}\right)=\left(\begin{array}{l}
S^{\Gamma} \\
0
\end{array}\right),
$$

where $\psi^{\Gamma(X)}$ - electrons wave function in $\Gamma(\mathrm{X})$-channel, $E$ - electrons energy, $H^{\Gamma(\mathrm{X})}$ 
-isolated $\Gamma(\mathrm{X})$-channel Hamiltonian, $\Sigma^{\Gamma(\mathrm{X})}$ operator of electrons extraction from $\Gamma(\mathrm{X})$ channel, $S^{\Gamma}$ - electron injection into $\Gamma$-channel function [18], $A$-coupling between channels operator.

The definition of a communication operator presented in [9] is not suitable for the case of a blurred aluminum profile, since it requires given coordinates of heterointerfaces; therefore, this definition is generalized to the case of a continuous aluminum profile.

$$
A=\alpha\left|\frac{d}{d z} x\right|,
$$

where $\alpha$-inter-valley interreaction constant, $x$-aluminium molar fraction, $z$ - coordinate.

The optical potential is included in the Hamiltonian of the $\Gamma$-channel and is assumed to be zero in spacer regions and barriers and a constant in quantum wells (in this work the value $0.02 \mathrm{eV}$ was used), thus the model includes an additional mechanism for the release of electrons from the quantum well. Again, such an optical potential distribution is not suitable for use in the case of the absence of explicit heterointerfaces. To take into account the effect of structure degradation on inelastic scattering processes, diffusion blurring of the optical potential profile was simulated using equations describing the diffusion of aluminum. The distribution of the optical potential as a sum of rectangular functions was used as the initial condition.

\section{SELF-CONSISTENT POTENTIAL}

The high time complexity of calculating the self-consistent potential (described in [11]) is associated primarily with the calculation of the concentration of electrons by formulaw

$$
\begin{aligned}
& n_{L(R)}=\frac{\sqrt{2} k T}{(2 \pi)^{2} \hbar^{3}}\left(m^{*}\right)^{\frac{3}{2}} \times \\
& \times \int_{U_{L(R)}}^{\infty}\left|\psi_{L(R)}\right|^{2} \ln \left(1+e^{\frac{-E+E_{F}+U_{L(R)}}{k T}}\right) \frac{1}{\sqrt{E-U_{l(r)}}} d E, \\
& (H-E+\Sigma) \psi_{L(R)}=S_{L(R)}, \\
& n=n_{L}+n_{R},
\end{aligned}
$$

where $n$ - electrons concentration, $k-$ Boltzmann constant, $T$ - temperature, $m^{*}$ - electrons in reservoirs effective mass, $\hbar$ Dirac constant, $U_{L(R)}-$ electron in source (drain) potential energy, $U_{l(r)}-$ electron potential energy at channel boundary with source (drain), $S_{L(R)}$ - electrons injection from source (drain) function, $E_{F}-$ Fermi level, $\varphi-$ envelope electrons wave function.

As can be seen from the above formulas the calculation of the electron concentration in the channel requires a separate calculation of the electron concentration from the source and drain, for which it is necessary to solve the Schrödinger equation twice, and then to calculate the integral in formulas (3) two times. Integration is complicated by the fact that the wave function has a number of narrow peaks (see Fig. 3) therefore, it is required to use multiple calculations with decreasing grid step or grid adaptation procedure.

Because $\psi_{L}$ and $\psi_{R}$ have resonances at the same energy levels, to reduce the computation time, you can use the same grid to calculate $n_{L}$ and $n_{\mathrm{R}}$. However, it is possible to propose a method that does not require the calculation of partial concentrations. By simple algebraic calculations, their formulas (3) can be obtained 

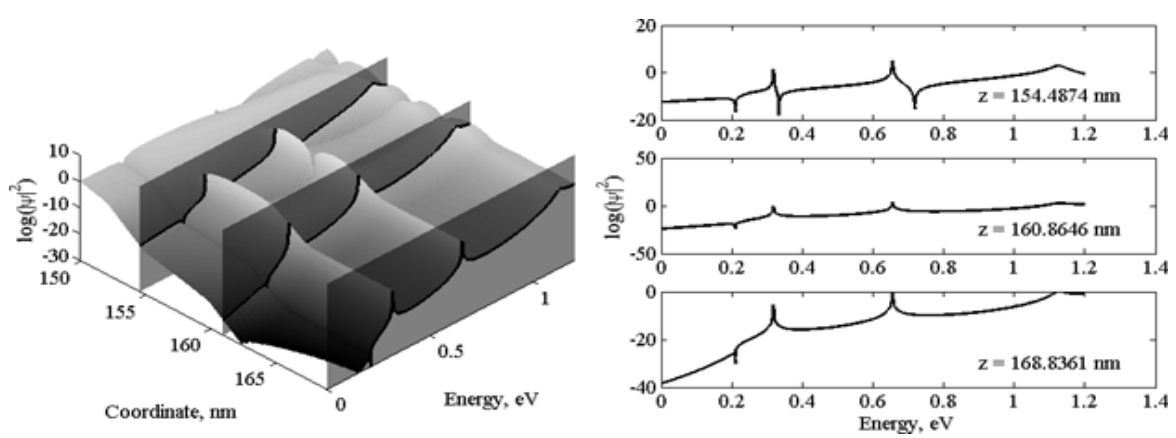

Fig. 3. The natural logarithm of the square of the modulus of the wave function of electrons from the source for fixed values of the coordinate.

$n=\frac{\sqrt{2} k T\left(m^{*}\right)^{\frac{3}{2}}}{(2 \pi)^{2} \hbar^{3}} \int_{U_{L}}^{\infty} \varphi d E$,

$(H-E+\Sigma) \varphi=S_{L} f_{L}+S_{R} f_{R}$,

$\left|f_{L(R)}\right|^{2}=\left\{\begin{array}{cc}\ln \left(1+e^{\frac{-E+E_{F}+U_{L(R)}}{k T}}\right) \frac{1}{\sqrt{E-U_{L(R)}}} \text { for } E>U_{L(R)} . \\ 0 & \text { for } E \leq U_{L(R)}\end{array}\right.$.

Calculating the electron concentration using formulas (4) allows solving the Schrödinger equation and integrating it once (for a given energy level), unlike calculating using formulas (3).

It is worth noting that if the Schrödinger equation for an open system [18] contains the functions of injection of electrons from the source $S_{L}$ and drain $S_{R}$, then in the continuity equation there appear interference components whose physicality is in question [14].

$$
d i \overline{v j}=\left|S_{L}\right|^{2}+\left|S_{L}\right|^{2}+S_{L} S_{R}^{*}+S_{L}^{*} S_{R},
$$

where $\bar{j}$ - probability flow.

As a result of numerical experiments, it was found that the contribution of interference terms to electrons density in spacers and potential barriers regions is negligible compared to the contribution of these terms to electrons density in potential well region. However, knowing the position of the potential well relative to the electron sources, it is possible to minimize the influence of interference terms by varying the arguments of complex $S_{L}$ and $S_{R}$, which is equivalent to the choice of the initial phases of the electrons wave functions.

The self-consistent potential was not significantly affected by interference terms. Thus, comparing the results of calculations of the self-consistent potential when using formulas (3) and (4) for calculating the concentration, no significant deviations were found (the maximum deviation is $\left.1.5 \cdot 10^{-5} \%\right)$. The results of calculating the self-consistent potential are shown in Fig. 4. The calculation time for various external voltages is presented in Fig. 5.

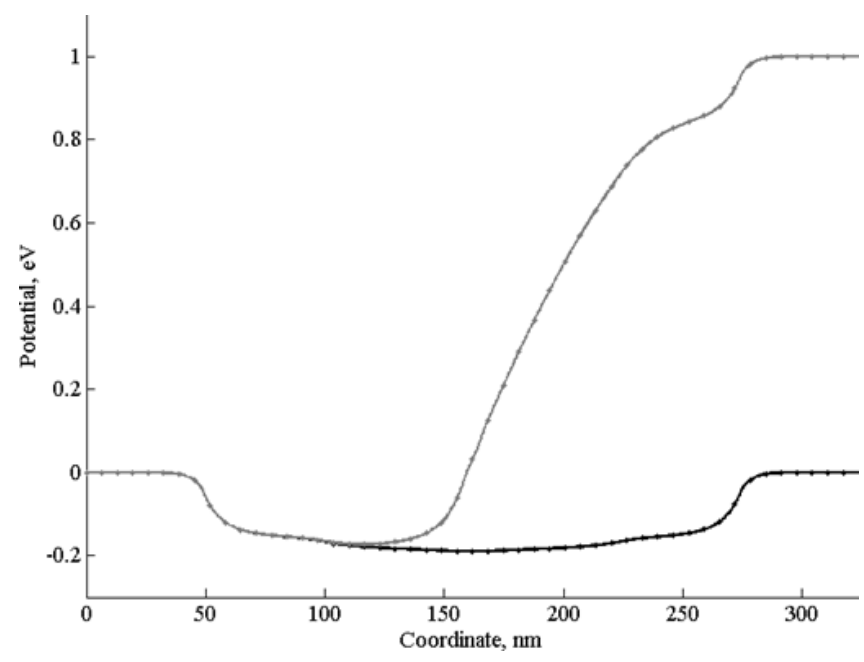

Fig. 4. Self-consistent potential at $O V$ (black) and $1 V$ (gray). Lines - the original algorithm, points an optimized algorithm. 


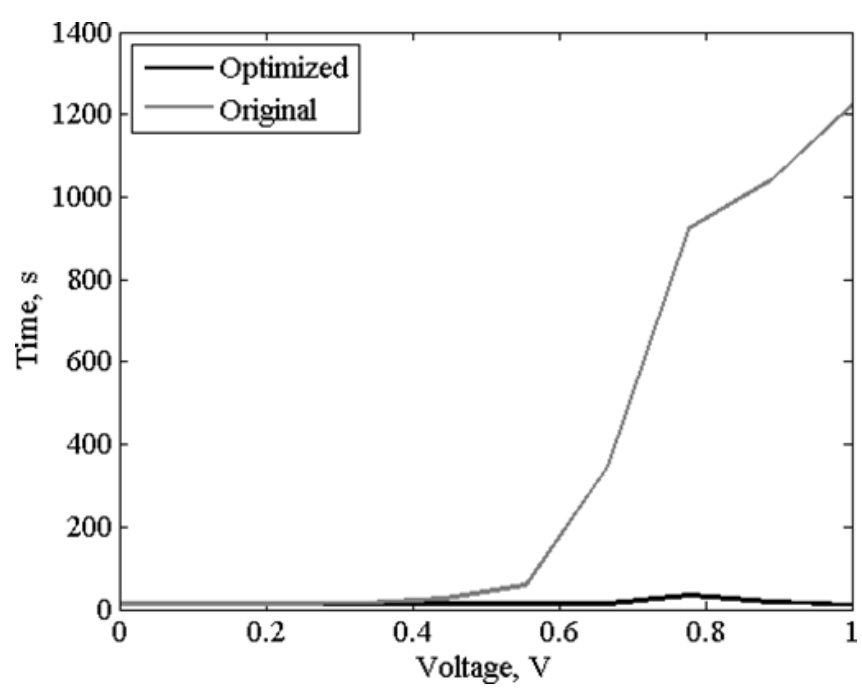

Fig. 5. Calculation time self-consistent potential.

Thus, the use of this approach made it possible to reduce the time for calculating the electron concentration by half without significant losses in accuracy.

\section{RESULTS}

For the validation of the presented model, the current-voltage characteristics of a number of RTS were calculated taking into account diffusion changes at the technological stage of annealing the structure for $30 s$ at $800^{\circ} \mathrm{C}$ [15-19]. The simulation was carried out self-consistently with regard to dissipative processes (the number of self-consistency iterations is 20, the inter-valley interaction constant is $0.7 \mathrm{eV} \AA$, the optical potential in the quantum well is $0.02 \mathrm{eV}$ ). The results of the calculation comapred with the experimental data are shown in Fig. 6.

As can be seen from the graphs, the developed algorithm allows, with satisfactory accuracy, to model the CVC of the RTS in the region of positive differential resistance - the deviation of the calculation results from the experimental data on the curvature of the initial portion does not exceed $3 \%$. Thus, the presented model can be used to predict the parameters of devices whose
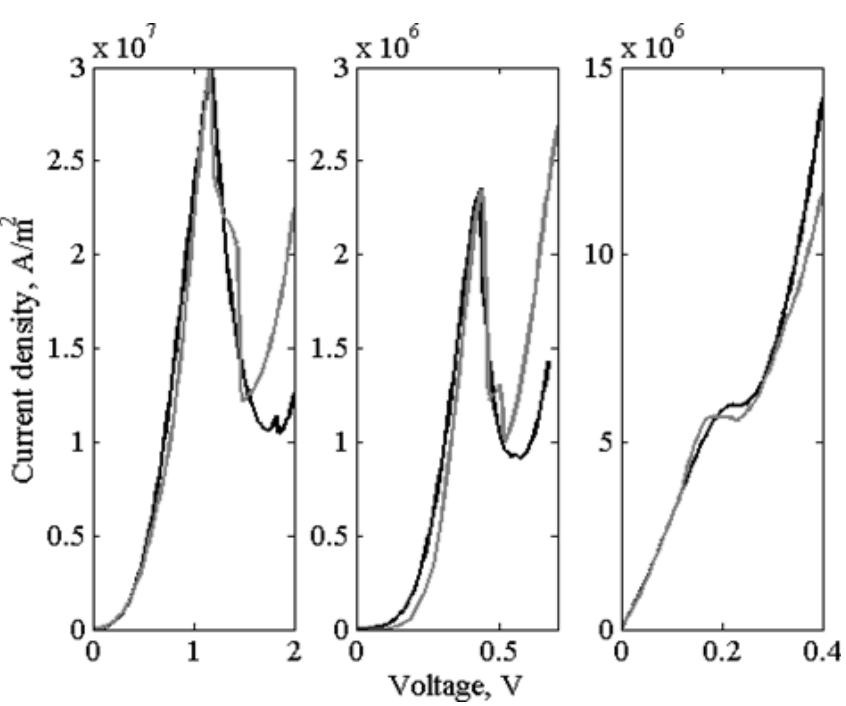

Fig. 3. The results of calculations IVC RTS. Grey lines - experimental data, black - the results of the calculation.

performance characteristics are determined by the initial section of the CVC RTS.

\section{CONCLUSION}

A mathematical model of the degradation of AlGaAs-heterostructures based on diffusion models of atoms in a crystal lattice has been developed. The diffusion of aluminum and silicon was simulated using the Fick equation with the second-type boundary conditions. For the numerical calculation, the finitedifference method was used, and the finitedifference scheme was built on the basis of a unified spatial grid for modeling both diffusion processes and transverse carrier transport, which made it possible to avoid loss of accuracy during interpolation. The blurring of the ohmic contacts was taken into account as an increase in the resistance of the contact layers. Accounting models of dissipative processes are generalized to the case of a continuous impurity profile. The operator of communication between the $\Gamma$ - and X-valleys was calculated on the basis of the aluminum fraction profile, thus, it was possible to take into account the effect of aluminum diffusion on the inter-valley 
scattering processes. In order to reduce the time complexity of the CVC calculation algorithm, the procedure for calculating the self-consistent potential is optimized at the stage of calculating the electron concentration by reducing the number of calculated integrals, thus, the time for calculating the self-consistent potential is reduced by half.

According to the results of validation of the presented model, it was concluded that it is useful to simulate the kinetics of the initial section of CVC of heterostructures at elevated temperatures, including within the framework of the task of predicting the GIC and MIC UHF reliability indicators based on multilayer AlGaAs-heterostructures.

\section{ACKNOwledgments}

This work was financially supported by the Ministry of Education and Science of the Russian Federation, project №16.1663.2017/4.6.

\section{REFERENCES}

1. Nadar S, Zaknoune M, Wallart X. High performance heterostructure low barrier diodes for sub-THz detection. IEEE Transactions on Terahertz. Science and Technology, 2017, 7(6):780-788.

2. Magruder KC, Levi AFJ. Optimal design of heterostructure tunnel diode with nonlinear current-voltage characteristic. Physica E, 2012, 44(7-8):1503-1509.

3. Bonsendorf D, Schneider S, Meinschien J, Tomm JW. Reliability of high power laser diodes with external optical feedback. Proc. of SPIE, 2016, 9733:973302.

4. Sin Y, Presser N, Lingley Z, Brodie M, Foran B, Moss S. Reliability, Failure Modes, and Degradation Mechanisms in High Power Singleand Multi-Mode
InGaAs-AlGaAs Strained Quantum Well Lasers. Proc. of SPIE, 2016, 9733:973304.

5. Lu Y, Nie Z, Zhang P, Wang Z, Xiong L Wang S, Wu D, Liu X. Numerical simulation of thermo-mechanical behavior in high power diode laser arrays. ICEPT, 2016:76-83.

6. Jimenez J. Laser diode reliability: crystal defects and degradation modes. Comptes Rendus Physique, 2003, 4(6):663-673.

7. Ushanov VI, Chaldyshev V, Preobrazhenskii VV, Putyato MA. Diffusion Blurring of GaAs Quantum Wells Grown at Low Temperature. Semiconductors, 2018, 52(13):1704-1707.

8. Makeev MO, Ivanov YA, Meshkov SA, Litvak YN, Vetrova NA. Issledovaniya degradatsii rezonansno-tunelnykh diodov na baze AlAs/GaAs nanogeterostructur [Studies of the degradation of resonant tunneling diodes based on AlAs/GaAs nanoheterostructures]. Inzhenerny zhurnal: nauka i innovatsii, 2013, 6(18):48 (in Russ.).

9. Abramov II. Osnovy modelirovaniya eblementov mikro- $i$ nanoeblektroniki [Fundamentals of modeling elements of micro- and nanoelectronics]. Saarbrücken, LAP LAMBERT Academic Publ., 2016, 444 p.

10. Bennabi Y, Hammou AB, Nouredine Z. Scattering and Tunneling in Active Systems. Mod. Phys. Lett. B, 2011, 25(20):1691-1700.

11. Wang J,Wasige E. Self-consistent analysis of the IV characteristics of resonant tunnelling diodes. Int. J. Terahertz Sci. Tech., 2012, 5(4):153-162.

12. Mennemann JF, Kosina H, Jungel A. J. Transient Schrodinger-Poisson Simulations of a High-Frequency 
Resonant Tunneling Diode Oscillator. Comput. Phys., 2013, 239:187-205.

13. Schowalter M. Investigation of diffusion in AlAs/GaAs distributed Bragg reflectors using HAADF STEM imaging. J. Phys.: Conf. Ser., 2011, 326:012035.

14. Kruglyak Y. Non-Equilibrium Green's Function Method in Matrix Representation and Model Transport Problems of Nanoelectronics. Nanomaterials: Applications And Properties, 2013, 2(1):01001(6pp).

15. Macherzynski W, GryglewiczJ, Stafiniak A, Prazmowska J, Paszkiewicz R. Formation Process and Properties of Ohmic Contacts Containing Molybdenum to $\mathrm{AlGaN} / \mathrm{GaN}$ Heterostructures. Advances in Electrical and Electronic Engineering, 2016, 14(1):83-88.

16. Piotrowska A. Ohmic Contacts to GaAs: Fundamentals and Practice. Acta Physica Polonica Series A, 1993, 84(3):491-504.

17. Nishi H, Inada T, Saito J, Ishikawa $\mathrm{T}$, Hiyamizu S. Implantation into an AlGaAs/GaAs Heterostructure. Jpn. J. Appl. Phys., 1983, 22:pp.401-404.

18. Yanxu Z., Weiwei C., Yuyu F., Ye D., Chen $\mathrm{X}$. Effects of rapid thermal annealing on ohmic contact of AlGaN/GaN HEMTs. Journal of Semiconductors, 2014, 35(2):6004.

19. Pillet CA. A Mathematical Account of the NEGF Formalism. Annales Henri Poincaré, 2018, 19(2):411-442. 